\title{
Programa de Promoção da Autorregulação da Aprendizagem de Ingressantes da Educação Superior
}

\author{
Fernanda Andrade de Freitas Salgado ${ }^{1}$ \\ Soely Aparecida Jorge Polydoro ${ }^{2}$ \\ Pedro Rosário ${ }^{3}$ \\ ${ }^{1}$ Centro Universitário Nossa Senhora do Patrocinio, Itú, SP \\ ${ }^{2}$ Universidade Estadual de Campinas, Campinas, SP \\ ${ }^{3}$ Universidade do Minho, Braga, Portugal
}

\begin{abstract}
Resumo
O objetivo deste trabalho foi avaliar a eficácia de parte de um programa para promover a autorregulação da aprendizagem na educação superior. Participaram 26 ingressantes com idade média de 20,65 (DP = 3,35) provenientes de diferentes cursos de graduação de uma universidade pública, os quais participaram de uma atividade não obrigatória, organizada em seis encontros de 90 minutos cada um. Medidas de autorrelato foram aplicadas no pré e pós-teste a fim de comparar as variáveis dependentes: conhecimento das estratégias de aprendizagem, processos de autorregulação da aprendizagem, instrumentalidade percebida, autoeficácia para autorregular-se e para a formação superior. De acordo com a comparação entre o pré e pós-teste, os resultados são favoráveis no pós-teste para as variáveis conhecimento das estratégias de aprendizagem, autorregulação da aprendizagem e autoeficácia para autorregular-se. Esses resultados sugerem que a autorregulação da aprendizagem de ingressantes pode ser potencializada por meio do programa educacional "Cartas do Gervásio ao seu Umbigo".

Palavras-chave: educação superior, autorregulação da aprendizagem, avaliação de programa educacional
\end{abstract}

Program to Promote Self-Regulated Learning in Higher Education Freshmen Students

\begin{abstract}
The objective of this study was to assess the efficacy of part of a program aimed at promoting self-regulation learning in higher education. Participants included 26 freshmen with mean age 20.65 (SD=3.35), from several undergraduate courses in a public university. The program was a non-mandatory activity organized in six 90-minute meetings. Self-report measures were applied in the pre and post test in order to compare the dependent variables, which were: knowledge about learning strategies, self-regulation learning strategies, perceived instrumentality, self-efficacy to self-regulate and self-efficacy for higher education. Data from pre and post tests indicate that the results are favorable in the post test for the variables knowledge about learning strategies, self-regulation learning strategies, and self-efficacy to self-regulate. These results suggest that self-regulated learning by freshmen may be increased by the program named "Letters from Gervásio to his navel".

Keywords: Higher education, sell-regulated learning; assessment of educational program
\end{abstract}

Programa de Promoción de Autorregulación del Aprendizaje de Ingresantes de la Educación Superior

\section{Resumen}

El objetivo de este trabajo fue evaluar la eficacia de parte de un programa, para promover la autoregulación del aprendizaje en la Educación Universitaria. El trabajo se realizó con 26 estudiantes con edad promedio entre 20,65 (DP=3,35) provenientes de diferentes cursos de graduación de una universidad pública, los cuales participaron de una actividad no obligatoria, organizada en seis encuentros de 90 minutos cada uno. Medidas de autoinforme fueron aplicadas en el pre y post test, a fin de comparar las variables dependientes: conocimiento de estrategias de aprendizaje, proceso de autoregulación de aprendizaje, instrumentalidad percibida, autoeficacia para autoregulación y para formación universitaria. De acuerdo con la comparación entre el pre y el post test, los resultados son favorables en el post test para las variables conocimiento de estrategias de aprendizaje, autoregulación de aprendizaje y autoeficacia para autoregulación. Estos resultados señalan que la autoregulación del aprendizaje de los ingresantes puede ser potencializada por medio del programa educacional "Cartas de Gervasio a su ombligo".

Palabras-clave: Educación Universitaria; autoregulación de aprendizaje; evaluación del programa educativo

A perspectiva da Teoria Social Cognitiva (TSC) considera o comportamento do sujeito originado na interação recíproca entre fatores pessoais, cognitivos e ambientais de maneira recíproca (Bandura, 1986; 2008). A relação de reciprocidade entre esses fatores varia a depender de cada pessoa e das circunstâncias (Bandura, 1986; 2008).
$\mathrm{Na}$ TSC, outro aspecto importante é a condição agêntica do sujeito, ou seja, o sujeito é capaz de exercer um papel ativo em sua própria história por meio de cinco capacidades eminentemente humanas, a saber, simbolização, intencionalidade, antecipação, autorregulação e autorreflexão, o que é postulado como agência pessoal (Bandura, 2001; 2008). Nesse estudo, destaca-se 
o construto da autorregulação o qual consiste no monitoramento, na avaliação e no controle pelo indivíduo de seu próprio comportamento e pensamento, em direção aos objetivos pessoais (Azzi \& Polydoro, 2017; Bandura, 2001; 2008).

No contexto educacional, Zimmerman (1989; 1998; 2000), tendo como referência os conceitos desenvolvidos por Albert Bandura, entende que a aprendizagem pode ser autorregulada à medida que $\mathrm{O}$ estudante estabeleça objetivos, que apresente crenças de confiança ao julgar sua capacidade para realizar determinada tarefa (autoeficácia), que se autoinstrua e conheça estratégias de aprendizagem. Esses processos ativados podem sustentar pensamentos, ações e afeto autogerados, os quais podem ser planejados e ciclicamente adaptados para atingir as metas acadêmicas (Zimmerman, 1989; 1998; 2000; Zimmerman \& Schunk, 2011), demarcando, assim, o papel agêntico que o estudante pode vir a ter ou deveria ter em seu processo de formação, ou seja, a capacidade de exercer papel ativo durante o seu processo formativo (Zimmerman, 1998).

Para Zimmerman (1998), a ARA é composta por três subprocessos: a fase prévia, a de realização e a de autorreflexão. A fase prévia do comportamento envolve a análise da tarefa e as crenças motivacionais. Para analisar a tarefa, pressupõe-se que o estudante, a partir do reconhecimento das atividades, de seus recursos pessoais e do ambiente, estabeleça metas e plano estratégico, tendo como base seu repertório de estratégias de aprendizagem, adaptando-as ao seu planejamento, às condições pessoais, comportamentais e ambientais. As crenças motivacionais influenciam o processo de antecipação das situações, seja com base nas crenças de autoeficácia, nas expectativas de resultados e nas crenças do valor intrínseco.

A fase de realização ocorre durante a execução da tarefa, por meio do autocontrole e da capacidade em auto-observar/automonitorar-se. $\mathrm{O}$ autocontrole pode ser alcançado por meio da autoinstrução; das representações mentais, auxiliadas pela autorrecordação; da atenção focada, para minimizar os distratores; e do uso de estratégias de aprendizagem. A auto-observação ocorre pelo automonitoramento do desempenho, das condições e das consequências obtidas (Zimmerman, 1998).

Já, a fase de autorreflexão envolve processos que ocorrem depois do esforço da aprendizagem e que, por sua vez, influenciam as reações do aprendiz, por meio do autojulgamento e da autorreação. $\mathrm{O}$ autojulgamento implica na comparação das informações advindas do automonitoramento com o padrão ou com as metas e a atribuição causal - autoavaliação (Zimmerman, 1998), o que sugere que o estudante pode reagir frente às comparações por duas razões: pela autossatisfação ou pelas inferências adaptativas (Zimmerman, 2000).

A autossatisfação envolve a percepção de satisfação e insatisfação do sujeito, a qual está ligada ao seu próprio desempenho, interferindo assim no estabelecimento de autoincentivos. No que se refere às inferências, as conclusões estabelecidas pelo sujeito após o seu esforço (alterar ou manter o comportamento) podem vir a alterar o enfoque de sua autorregulação, podendo ser adaptativas ou defensivas, como, por exemplo, a apatia e a procrastinação (Zimmerman, 2000). De forma sintética, essas autorreações do sujeito afetam os processos antecipatórios de maneira cíclica, interferindo em futuras ações e no estabelecimento de metas (Bandura, 1991; Schunk, 2001; Zimmerman, 1998), o que reforça o processo dinâmico da ARA.

Apesar da ênfase nos processos internos-autorregulatórios do sujeito, reconhece-se a influência dos processos externos - o ambiente, a exemplo da influência dos pais, professores, sistema educacional, bem como os comportamentos emitidos pelo sujeito e as influências recíprocas entre esses eventos (Zimmerman, 1989). Ou seja, os estudantes não são meros recipientes passivos de informação, pelo contrário, são capazes de exercer controle e regular seu processo de aprendizagem (Emílio \& Polydoro, 2017).

Promover os processos autoregulatórios de estudantes do ensino superior é alvo de diversos programas localizados na literatura estrangeira (Hattie, Biggs, \& Purdie, 1996, Rosário et al., 2007; Rosário, Mourão, Núñez, \& González-Pienda 2010a; Rosário, Nunes, Magalhães, Rodrigues, Pinto, \& Ferreira, 2010b; Rosário, Nunez, González-Pienda, Valle, Trigo, \& Guimarães, 2010c; Simpson, Hynd, Nist, \& Burrell, 1997). Tendo como foco a formação de professores, Fabriz, Ewijk, Poarch e Büttner (2014) realizaram uma investigação sobre a função de automonitoramento por meio do uso de diários, e Zimmerman, Bonner e Kovach (2008) descreveram uma proposta cujo objetivo era promover o gerenciamento de tempo, a compreensão textual, as anotações em aula, a preparação para os testes e para a escrita.

Esses programas também podem ser aplicados diretamente aos alunos. Khosa e Violet (2013) desenvolveram uma intervenção metacognitiva na disciplina de fisiologia no curso de Medicina Veterinária; e Schloemer e Brenan (2006) desenvolveram um curso com 
o propósito de trabalhar estabelecimento de metas, automonitoramento e estratégias de aprendizagem. Parres e Flores (2011) desenvolveram uma oficina com estudantes da licenciatura da área de ciências sociais e humanidades; Lan (1998) descreveu uma proposta de infusão curricular das estratégias metacognitivas em uma disciplina de estatística.

Já Seco, Pereira, Santos, Filipe e Alves (2008) descreveram um programa cujo objetivo era apoiar o desenvolvimento das capacidades para identificar e usar as estratégias de estudos mais adequadas para cada situação; Vanderstoep e Pintrich (2008), por sua vez, elaboraram um material em formato de livro que dizia respeito a como se tornar um aprendiz autorregulado, por meio do estabelecimento de metas, da motivação, do gerenciamento das fontes externas e internas, da atenção e da memória, da cognição e metacognição, das anotações, da leitura e escrita, da realização de provas, do pensamento crítico e da resolução de problemas. Hofer, Yu e Pintrich (1998) ofereceram um curso para ensinar estratégias autorregulatórias; e Sánchez (2004) desenvolveu um modelo de estratégia de leitura significativa de textos.

Dentre esse leque, selecionou-se o programa intitulado "Cartas do Gervásio ao seu umbigo: comprometer-se com o estudar na Educação Superior" (Rosário, Núñez, \& Gonzalez-Pienda, 2012), que tem a teoria social cognitiva como base teórica e o uso da narrativa para fomentar os processos autorregulatórios. A escolha por esse programa se justifica também pelos resultados indicarem boa aceitação e eficácia em diferentes países como a Espanha, Portugal, Moçambique, Chile e África do Sul (Cerezo, 2010; Magalhães, 2009; Nunes, 2009; Pina, Rosário, \& Tejada, 2008; Pina, Rosário, Tejada, \& Lara, 2006; Rosário et al., 2007; Rosário et al.; 2010a; Rosário et al., 2010b; Rosário et al, 2010c, Solano, 2006) e, no Brasil (Dantas, Pelissoni, Freitas-Salgado, Polydoro, \& Rosário, 2017; Freitas-Salgado, 2013; Magalhães, 2012; Pelissoni, 2016; Rosário, Polydoro, Fuentes, \& Gaeta, 2012; Rosário, \& Polydoro, 2014).

Segundo os resultados de algumas dessas pesquisas, os alunos tendem a apresentar melhores resultados a favor do pós-teste, diminuição no processo superficial de estudo, maior conhecimento das estratégias de aprendizagem, tendendo a planejar, executar e avaliar mais as atividades de estudo (Cerezo, 2010; Freitas-Salgado, 2013; Magalhães, 2009; Nunes, 2009; Pelissoni, 2016; Pina, Rosário, \& Tejada, 2008; Pina, Rosário, Tejada, \& Lara, 2006; Rosário et al., 2007; Rosário et al.; 2010a; Rosário et al., 2010b; Rosário et al., 2010c,
Rosário et al., 2012; Rosário, \& Polydoro, 2014; Solano, 2006).

O programa fundamenta-se nos processos de ARA de Zimmerman (2000; 2002), os quais Rosário, Núñez e González-Pienda (2007) apresentam-na, de forma sintética, por meio de três fases, a saber, $\mathrm{Pla}$ nejar (Pl), Executar(E) e Avaliar(A) (PLEA) a fim de desenvolver o programa "Cartas do Gervásio ao seu umbigo". Além desse suporte teórico, esse programa tem também como pilar o processo de modelação (Rosário et al., 2012; Rosário, \& Polydoro, 2014) que significa a aprendizagem por meio de modelos, a qual está subordinada a quatro subprocessos inter-relacionados: atenção, retenção ou lembrança do comportamento, reprodução motora e o reforço, e motivação (Bandura, 1986) que pode afetar pensamentos, crenças e/ou comportamentos (Schunk, 2001).

Nesse caso, Gervásio e o seu umbigo, e outros personagens nas estórias do Programa servem de modelos, a fim de ensinar estratégias e o automonitoramento. Além desses personagens, professores, pares e outros adultos podem ser modelos influentes para os alunos (Rosário \& Polydoro, 2014). O protagonista das estórias do Programa é o estudante Gervásio que, à medida que escreve suas cartas, além de relatar suas experiências pessoais, interpessoais, institucionais e de estudo, elabora suas vivências a partir de reflexões, questionamentos e orientações provocadas, em especial, pelo umbigo - metáfora da autorreflexão -, mas também decorrente da interlocução com colegas e professores (Rosário et al., 2012).

Cada carta tem como proposta implementar uma ou mais estratégias de aprendizagem. $\mathrm{Na}$ fase do Planejamento, as estratégias são: estabelecimento de objetivos, autoavaliação e busca de ajuda. A fase de Execução inclui estratégias de organização, gestão do tempo, anotações, listas de coisas a fazer, resolução de problemas e memória. Na fase da Avaliação, encontram-se estratégias de autorreflexão e autoconsequências (Rosário et al., 2012). A partir dos pressupostos teóricos associados às peculiaridades do programa em questão, procurou-se avaliar a eficácia de parte do programa "Cartas do Gervásio ao seu umbigo" no formato de Oficina para ingressantes em uma universidade pública brasileira.

\section{Método}

Para aferir a eficácia do programa "Cartas do Gervásio ao seu umbigo", foi desenvolvida uma pesquisa cujo delineamento foi o quase experimental. Tomando 
as categorias de Campbell e Stanley (1979), o delineamento foi o de série temporal.

\section{Participantes}

A composição atendeu ao critério de conveniência, a partir da inscrição espontânea do aluno ao programa. Para o estudo, foram considerados os 26 ingressantes que estiveram presentes na totalidade dos encontros. Esses tinham idade média de 20,65 anos $(D P=3,35)$ variando de 17 a 29 anos de idade, sendo a maioria composta por homens $(n=17 ; 65,4 \%)$ e a minoria por mulheres $(n=9 ; 34,6 \%)$, pertencentes a cursos da área de Ciências Exatas, Tecnológicas e da Terra $(n=19$; $73,1 \%)$, Ciências Humanas $(n=5 ; 19,2 \%)$ e Artes $(n$ $=2 ; 7,7 \%)$ e frequentavam o turno integral $(n=22$; $84,6 \%)$ e matutino $(n=4 ; 15,4 \%)$.

\section{Instrumentos}

a) Ficha de Identificação: composta por itens sobre características pessoais e de informações acadêmicas.

b) Questionário de Conhecimentos de Estratégias de Aprendizagem (CEA) (Pina, Rosário, Tejada, \& Lara 2006): É uma medida de desempenho que tem como objetivo avaliar os conhecimentos dos alunos acerca das estratégias de aprendizagem, o qual é composto de dez questões com três opções de respostas, sendo duas falsas e uma verdadeira; a pontuação obtida corresponde à soma de respostas corretas. Os itens referem-se às estratégias: cognitivas (cinco itens), metacognitivas (dois itens), motivacionais (um item), gestão de recursos e busca de ajuda (dois itens). Essa medida foi adaptada para o contexto nacional com índices de consistência interna de alfa de Cronbach de 0,87 (Polydoro, Rosário, Sampaio, \& Freitas, 2011).

c) Inventário de Processos de Autorregulação da Aprendizagem - Universidade (IPAA) (Rosário, 2009a): trata-se de medida de autorrelato que tem como objetivo avaliar a frequência de processos autorregulatórios (planejamento, execução e avaliação). Originalmente é composto por nove itens para serem respondidos tendo como base uma escala Likert, de cinco pontos: 1 (nunca) a 5 (sempre). A pontuação obtida corresponde ao somatório das respostas dadas e dividida pelo total de itens. $\mathrm{O}$ instrumento foi adaptado e validado para o contexto brasileiro e, em estudo preliminar, obteve consistência interna por meio do alfa de Cronbach de 0,75 (Polydoro et al., 2011) próximo aos obtidos por Rosário et al. (2007), Magalhães (2009) e Nunes (2009) em que o alfa de Cronbach encontrado foi de 0,87 .

d) Questionário de Instrumentalidade Percebida para Autorregular a Aprendizagem (QIAR) (Rosário, 2009b): de origem portuguesa, é uma medida de autorrelato que tem como objetivo avaliar a frequência da percepção dos estudantes acerca da utilidade das estratégias de aprendizagem para autorregular sua aprendizagem e é composto por dez afirmações, sendo que, para cada uma, o aluno responde em que medida a considera útil, tendo como base uma escala Likert: 1 (nada úti) a 5 (muito útil). A pontuação obtida corresponde ao somatório das respostas dividida pelo número total de itens. O instrumento foi adaptado e validado para o contexto brasileiro e, em estudo preliminar, obteve consistência interna aferida pelo alfa de Cronbach de 0,79 (Polydoro et al., 2011). No estudo de Nunes (2009), o alfa de Cronbach obtido foi de 0,87 .

e) Questionário de Autoeficácia para Autorregular-se (QAEAR) (Rosário, 2009b): de origem portuguesa, trata-se de medida de autorrelato que tem como objetivo mensurar a frequência da percepção do estudante sobre sua capacidade de autorregular sua aprendizagem. Originariamente é composto pelas mesmas dez afirmações do instrumento anterior, sendo que, para cada uma, o aluno responde em que medida se sente capaz de utilizá-la, tendo como base uma escala Likert: 1 (nada capas) a 5 (muito capaz). A pontuação obtida corresponde à somatória das respostas dividida pelo total de itens. $\mathrm{O}$ instrumento foi adaptado e validado para o contexto brasileiro e, em estudo preliminar, obteve consistência interna avaliada pelo alfa de Cronbach de 0,81 (Polydoro et al., 2011). Já no estudo de Nunes (2009), o alfa de Cronbach foi de 0,91 .

f) Escala de Autoeficácia para a Formação Superior (AEFS) (Polydoro \& Guerreiro-Casanova, 2010): Trata-se de uma medida de autorrelato que tem como objetivo aferir a frequência da percepção sobre a capacidade do estudante organizar e executar cursos de ações requeridos para produzir certas realizações referentes aos aspectos compreendidos pelas vivências acadêmicas pertinentes ao ensino 
superior (Polydoro \& Guerreiro-Casanova, 2010). Essa escala é composta por 34 questões que podem ser respondidas por meio de uma escala Likert de 10 pontos: 1 (pouco capaz) e 10 (muito capas). A pontuação foi obtida por meio da somatória das respostas aos itens de cada dimensão e para a escala como um todo dividida pela quantidade de itens constitutivos. Polydoro e Guerreiro-Casanova (2010) descrevem que a escala total apresenta índice de consistência interna de 0,94 , e que os cinco fatores foram: autoeficácia (AE) acadêmica, AE na regulação da formação, AE na interação social, AE nas ações proativas e AE na gestão acadêmica. Os índices de consistência interna desses fatores variaram de 0,80 a 0,87 .

g) Programa “Cartas do Gervásio ao seu umbigo: comprometer-se com o estudar na universidade" (Rosário; Núñez, \& González-Pienda, 2012): Esse programa foi a base para a manipulação da variável independente ARA por meio de algumas estratégias autorregulatórias. Para tanto, foram selecionadas seis cartas do Programa para a presente intervenção (Figura 1).

A escolha das cartas foi norteada com base em resultados de pesquisas que descreviam as dificuldades de ingressantes na educação superior. Além disso, os temas como estratégias autorregulatórias, anotações, gerenciamento do tempo e estabelecimento de metas escolhidos também são focos de programas promovidos por Hofer, Yu e Pintrich (1998), Schloemer e Brenan (2006), Seco et al. (2008), Vanderstoep e Pintrich (2008) e Zimmerman, Bonner e Kovach (2008).

a) Avaliação da oficina: composta no total de cinco itens. Os itens diziam respeito à percepção da relevância das estratégias autorregulatórias bem como sobre o impacto dos conteúdos abordados na vida acadêmica, além de espaços para sugestões e observações gerais.

\section{Procedimento de Coleta de Dados}

Após aprovação do projeto pelo Comitê de Ética em Pesquisa (CEP), organizou-se a coleta de dados, que ocorreu no segundo semestre de 2010, como atividade do serviço de apoio ao estudante da instituição. O grupo foi composto a partir dos estudantes que se inscreveram on-line na oficina "Como estudar melhor agora que estou na Universidade?", divulgada por meio da página virtual do serviço, e-mails institucionais, cartazes e folhetos e consentiram a sua participação mediante assinatura do Termo de Consentimento Livre e Esclarecido. A oficina teve início em meados de setembro e foi finalizada no início do mês de novembro de 2010.

A aplicação do pré e pós-teste foi coletiva, com duração média de 25 minutos, e ocorreram no primeiro e último dia da oficina, respectivamente. As medidas de autorrelato - a saber, o CEA, QIAR, QAEAR, IPAA e o AEFS -, foram aplicadas tanto no pré como no pós-teste. $\mathrm{O}$ instrumento para aferir o impacto na perspectiva dos participantes foi aplicado no último encontro junto com o pós-teste.

As atividades do programa ocorreram em encontros com duração de 90 minutos, realizados semanalmente, de forma ininterrupta, totalizando seis encontros. Foram padronizados em termos de sequência de atividades, materiais utilizados, presença de uma auxiliar e da coordenadora. A auxiliar, pesquisadora da teoria social cognitiva, contribuía com a entrega e recolha dos materiais, orientação e acompanhamento de alguns subgrupos, além de oferecer uma síntese do ocorrido ao final de cada encontro. O procedimento de cada encontro ocorreu, de forma geral (Figura 1), conforme sugestão dos autores do Programa. As etapas são: leitura da carta, discussão em subgrupos (socialização de dúvidas e/ou vivências dos próprios alunos) disparada por alguma atividade direcionada, socialização em plenária e síntese feita pela coordenadora sobre o tema tratado (Rosário; Núñez, \& González-Pienda, 2012; Freitas-Salgado, 2013). No momento "leitura da carta", a carta escolhida por encontro era lida. Dessa forma, as seis cartas utilizadas, serão mencionadas tal como foram apresentadas aos estudantes: primeiro encontro $=$ carta 0 ; segundo encontro $=$ carta 6 ; terceiro encontro $=$ carta 2 ; quarto encontro $=$ carta 4 ; quinto encontro $=$ carta 3 ; sexto encontro $=\operatorname{carta} 13($ Figura 1$)$.

\section{Procedimentos de Análise dos Dados}

Para a realização da análise quantitativa, os dados foram submetidos ao programa Statistical Analysis System (SAS, 2001). Os resultados não se adequaram à distribuição normal e o nível de significância adotado para os testes estatísticos foi de $5 \%$, ou seja, $p<$ 0,05 . Já a análise qualitativa foi realizada com base nas respostas dadas pelos participantes ao instrumento denominado "Avaliação da oficina" por meio da análise de conteúdo. 


\begin{tabular}{|c|c|c|}
\hline Encontro & Carta do Programa & Etapas \\
\hline $1^{\mathrm{o}}$ & $\begin{array}{l}\text { Carta } 0=\text { Se lerem as cartas com atenção, } \\
\text { poderão entender os sinuosos contornos } \\
\text { da minha experiência como calouro na } \\
\text { universidade e testemunhar comigo o } \\
\text { acontecido. }\end{array}$ & $\begin{array}{l}\text { 1. Explicação do objetivo do encontro, } \\
\text { 2. Aplicar o TCLE + Pré-teste } \\
\text { 3. Dinâmica de apresentação, } \\
\text { 4. Leitura da carta. } \\
\text { 5. Discussão em subgrupos, } \\
\text { 6. Socialização com todo o grupo, } \\
\text { 7. Sumário dos aspectos apontados. } \\
\text { 8. Encerramento }\end{array}$ \\
\hline $2^{\circ}$ & $\begin{array}{l}6=\text { Quem governa a sua aprendizagem, } \\
\text { Gervásio? Você sabe como se distinguem os } \\
\text { alunos que obtêm sucesso escolar? }\end{array}$ & $\begin{array}{l}\text { 1. Explicação do objetivo do encontro, } \\
\text { 2. Leitura da carta. } \\
\text { 3. Requisição de uma atividade prática } \\
\text { 4. Sumário dos aspectos apontados. } \\
\text { 5. Encerramento }\end{array}$ \\
\hline $3^{\circ}$ & $\begin{array}{l}2=\text { Que objetivos tenho? O que é que } \\
\text { verdadeiramente guia o meu agir no meu } \\
\text { estudo, na universidade, nos meus hobbies, } \\
\text { na prática de esportes, nas relações com os } \\
\text { outros, na minha preguiça...? }\end{array}$ & $\begin{array}{l}\text { 1. Explicação do objetivo do encontro, } \\
\text { 2. Dinamica de grupo - dividir subgrupos, } \\
\text { 3. Leitura da carta. } \\
\text { 4. Requisição de uma atividade prática, } \\
\text { 5. Solicitação da principal mensagem do encontro } \\
\text { 6. Sumário dos aspectos apontados. } \\
\text { 7. Encerramento }\end{array}$ \\
\hline $4^{\circ}$ & $\begin{array}{l}4=\text { Você sabe como vencer a } \\
\text { procrastinação, Gervásio? }\end{array}$ & $\begin{array}{l}\text { 1. Eexplicação do objetivo do encontro, } \\
\text { 2. Requisição de uma atividade reflexiva, } \\
\text { 3. Leitura da carta } \\
\text { 4. Requisição de uma atividade reflexiva } \\
\text { 5. Socialização com o grupo } \\
\text { 6. Requisição de uma atividade reflexiva } \\
\text { 7. Sumário dos aspectos apontados. } \\
\text { 8. Encerramento }\end{array}$ \\
\hline $5^{\circ}$ & $\begin{array}{l}3 \text { = Como posso realizar melhores } \\
\text { anotações? }\end{array}$ & $\begin{array}{l}\text { 1. Explicação do objetivo do encontro, } \\
\text { 2. Formação de subgrupos por área de conhecimento, } \\
\text { 3. Leitura da carta } \\
\text { 4. Requisição de uma atividade reflexiva } \\
\text { 5. Socialização com o grupo } \\
\text { 6. Requisição de uma atividade reflexiva } \\
\text { 7. Sumário dos aspectos apontados. } \\
\text { 8. Encerramento }\end{array}$ \\
\hline $6^{\circ}$ & 13 = Como vai o seu estudo, Gervásio? & $\begin{array}{l}\text { 1. Explicação do objetivo do encontro, } \\
\text { 2. Leitura da carta } \\
\text { 3. Requisição de uma atividade reflexiva } \\
\text { 4. Aplicação do pós-teste e avaliação da oficina } \\
\text { 5. Entrega dos certificados } \\
\text { 6. Encerramento }\end{array}$ \\
\hline
\end{tabular}

Figura 1. Descrição do procedimento e das cartas do programa "Cartas do Gervásio ao seu umbigo" por encontros. 


\section{Resultados e Discussão}

Os resultados obtidos por meio das medidas de autorrelato antes e depois da intervenção, bem como o resultado do teste de $W$ ilcoxon estão expressos na Tabela 1. De forma sintética, o teste de Wilcoxon sinaliza que houve diferenças significantes a favor do pós-teste em todas as variáveis dependentes.

Os resultados obtidos quanto às variáveis conhecimento de estratégias de aprendizagem, percepção da frequência de comportamentos autorregulatórios adotados nas tarefas de aprendizagem, percepção de autoeficácia para autorregular-se são convergentes com os de outros estudos que utilizaram o Programa, como Cerezo (2010), Magalhães (2009), Nunes (2009), Pelissoni (2016), Pina et al. (2006), Pina, Rosário e Tejada (2008), Rosário et al. (2007), Rosário et al. (2010a); Rosário et al. (2010b); Rosário et al. (2010c), Solano (2006). Ainda, o estudo de Pelissoni (2016) também observou que a percepção de autoeficácia na formação superior no total e em suas dimensões - a saber, AE acadêmica, AE na regulação da formação, $\mathrm{AE}$ na interação social, $\mathrm{AE}$ nas ações proativas e $\mathrm{AE}$ na gestão acadêmica -, foram significativamente maior a favor do pós-teste.

Os sujeitos desta pesquisa escolheram participar da intervenção de uma atividade não obrigatória de forma voluntária. Esse dado sugere que as condições de escolha são importantes fontes de informação uma vez que é possível observar o papel agêntico do estudante (Bandura, 2001; 2008) e por saber que as crenças motivacionais influenciam, em parte, nos processos da ARA, seja no estabelecimento de objetivos e na escolha/uso das estratégias de aprendizagem (Bandura, 2001; 2008; Pajares, \& Olaz, 2008; Rosário, 2004; Zimmerman, 2000).

Outro fator de impacto pode ser o fato de os participantes encontrarem pessoas reais ou fictícias, que partilhavam de dificuldades ou reflexões parecidas seja por meio da leitura da carta como na discussão dos subgrupos. Esse compartilhamento permitia a ampliação de conhecimento de estratégias bem como legitimava recursos já utilizados pelos estudantes, garantindo assim mais opções de escolha diante de uma tarefa acadêmica. Entende-se que algumas fontes de autoeficácia, nesse caso, as experiências vicárias, melhor dizendo, aprendizagem por meio de observações de modelos simbólicos e/ou reais e a persuasão social, ou seja, expor os estudantes a avaliações verbais de terceiros (Pajares, \& Olaz, 2008) podem justificar o aumento da autopercepção na sua capacidade de estabelecer metas, fazer escolhas, planejar e autorregular suas ações no processo de formação e desenvolvimento de carreira.

A relação entre a variável independente da pesquisa (ARA) e a variável dependente, crenças de AE, pode ser justificada uma vez que essas interferem no processo de autorregulação. Ou seja, as crenças de AE estão associadas ao estabelecimento de objetivos, à seleção das estratégias, além de ajudar a determinar quanto de esforço os alunos dedicarão à tarefa e à perseverança diante dos obstáculos (Pajares \& Olaz, 2008; Rosário, 2004).

Tabela 1

Estatística Descritiva das Variáveis Dependentes e Comparação Intragrupo segundo o Teste Wilcoxon

\begin{tabular}{|c|c|c|c|c|c|c|c|}
\hline \multirow{2}{*}{ Variáveis } & \multicolumn{3}{|c|}{ pré-teste } & \multicolumn{4}{|c|}{ pós-teste } \\
\hline & Mediana & $M$ & $D P$ & Mediana & $M$ & $D P$ & $p$ \\
\hline Conhecimento das Estratégias de aprendizagem (CEA) & 9,00 & 7,92 & 2,01 & 9,50 & 9,07 & 1,49 & 0,003 \\
\hline Autorregulação da aprendizagem (IPAA) & 3,56 & 3,55 & 0,57 & 4,00 & 3,87 & 0,56 & 0,004 \\
\hline Instrumentalidade para autorregulação (QIAR) & 4,67 & 4,55 & 0,39 & 4,78 & 4,73 & 0,25 & 0,002 \\
\hline Autoeficácia para autorregular-se (QAEAR) & 3,00 & 3,17 & 0,64 & 3,78 & 3,79 & 0,58 & $<0,001$ \\
\hline Autoeficácia na formação superior (AEFS) & 6,37 & 6,80 & 1,33 & 7,71 & 7,70 & 1,33 & $<0,001$ \\
\hline Autoeficácia acadêmica & 6,56 & 6,90 & 1,24 & 8,11 & 7,87 & 1,35 & $<0,001$ \\
\hline Autoeficácia na regulação da formação & 6,50 & 6,64 & 1,74 & 7,57 & 7,46 & 1,67 & 0,014 \\
\hline Autoeficácia na interação social & 6,86 & 6,79 & 1,63 & 7,71 & 7,73 & 1,32 & 0,001 \\
\hline Autoeficácia nas ações proativas & 6,14 & 6,48 & 1,57 & 7,50 & 7,32 & 1,52 & 0,004 \\
\hline Autoeficácia na gestão acadêmica & 7,13 & 6,94 & 1,63 & 8,13 & 7,86 & 1,56 & 0,002 \\
\hline
\end{tabular}


Por outro lado, os dados sobre a variável instrumentalidade percebida aferida pelo QIAR, divergiram dos resultados de estudos desenvolvidos por outros autores (Cerezo, 2010; Nunes, 2009; Rosário et al., 2007; Rosário et al., 2010c; Solano, 2006), já que esses indicaram não ter havido diferenças significantes entre o pré e o pós-teste. A diferença a favor do pós-teste sugere que a oficina pode ter reafirmado a importância de algumas estratégias autorregulatórias no processo de aprendizagem.

Faz-se relevante sinalizar que a média foi consideravelmente alta no pré-teste para a variável QIAR e CEA (Tabela 1), o que sugere que os ingressantes já conhecem e percebem a utilidade de algumas estratégias autorregulatórias. Sabe-se que esses ingressantes apresentam histórico escolar de investir muitas horas de estudo a fim de disputar uma vaga nos vestibulares brasileiros e/ou obter boa nota no Enem (Exame nacional do ensino médio) para ingressarem numa instituição de ensino superior de sua preferência.

Todavia, acredita-se que a modelação ativada pela leitura das cartas pertencentes ao programa, a troca entre os pares nos espaços disponibilizados para discussão e realização de alguma atividade reflexiva ou prática, ou seja, por meio de experiências diretas, a persuasão social, por meio da descrição de alguma estratégia autorregulatória por parte da coordenadora, relato de experiências vividas pelos pares-estudantes e, por meio da síntese realizada ao final de cada encontro, foram fatores que contribuíram para o maior conhecimento de estratégias de aprendizagem, o fortalecimento da percepção de sua capacidade para usar e autorregular estratégias de aprendizagem, bem como da importância dessas para otimização do processo de aprendizagem, semelhantes aos dados obtidos por Pelissoni (2016) com estudantes brasileiros. Além disso, reconhecer que algumas etapas para promoção da ARA são importantes, seja para promover a modelação/observação, guiar a realização de alguma técnica/estratégia-prática guiada, permitindo que aconteça a interiorização das estratégias para que a prática autônoma seja possível (Zimmerman, 2000; Rosário, 2004).

Conforme as respostas dadas pelos estudantes às questões abertas sobre o impacto da oficina no seu processo de aprendizagem e estudo, cinco categorias de mudança foram estabelecidas. Cada uma delas serão descritas a seguir: 1 . Incremento de motivação e segurança pessoal; 2. Aumento do conhecimento e da percepção de instrumentalidade; 3. Autopercepção e identificação de estratégias de enfrentamento; 4. Maior apropriação do ambiente universitário; e 5. Fortalecimento do processo de ARA.

Em relação à categoria observada "Incremento de motivação e segurança pessoal", pode-se afirmar que os estudantes descreveram que se tornaram mais confiantes em si mesmos para promover mudanças e se perceberam mais à vontade para estudar, assim como mais seguros para entender a matéria. A fim de justificar essa categoria, dois relatos dos estudantes são exibidos: "[...] a oficina me ensinou a ver algum sentido e tomar algum gosto pelas matérias que classifico como chatas" (A., Química) e "Com a oficina [...] me sinto mais confiante em fazer uma mudança efetiva no estudo" (B, Física/Matemática/Matemática Aplicada e Computacional).

A categoria "Aumento do conhecimento declarativo e da percepção da instrumentalidade" refere-se à absorção de informações sobre como melhorar o estudo a partir do aprendizado e/ou aperfeiçoamento de estratégias de aprendizagem e da identificação da utilidade dessas estratégias. Para exemplificar essa categoria seguem falas de estudantes: "A oficina proporcionou diversas reflexões e mostrou de várias formas como o estudo pode ser melhorado [...]" (C., Física/ Matemática/Matemática Aplicada e Computacional) e "[...] compreendi a importância das estratégias e vou tomá-las como referência para definir como estudarei daqui em diante" (D., Física/ Matemática/Matemática Aplicada e Computacional).

Quanto à categoria Autopercepção e identificação de estratégias de enfrentamento, esta diz respeito à realização de autoavaliação diante das questões de estudo, identificação de pontos fracos e limitações, bem como a busca de soluções, por exemplo, alterar a estratégia de aprendizagem utilizada. Para ilustração seguem relatos de estudantes: "[...] com o passar das sessões fui percebendo onde eu acertava e onde poderia melhorar, com isso pude me organizar melhor e tornar o meu tempo mais produtivo e proveitoso" (E., Engenharia Elétrica) e "[...] a oficina me deu ferramentas para rever minhas estratégias de aprendizagem, me mostrando o caminho para consertá-las [...] Foi a partir de então que pude verificar quais estavam sendo meus erros e a partir de então elaborar novas estratégias para tal" (F., Pedagogia).

$\mathrm{Na}$ categoria Maior apropriação do ambiente universitário, considerou-se o relato dos estudantes sobre a contribuição da oficina para sua integração às novas exigências e à rotina da universidade. Estudantes relataram que "Acredito que minha participação na oficina foi importante para me situar no ambiente acadêmico 
e passar, a saber, lidar com as situações que enfrentarei [...]" (G., Engenharia Elétrica) e "A oficina me ajudou bastante a adaptar a minha forma de estudar as exigências da universidade" (H., Estatística).

Por fim, no que corresponde à categoria Fortalecimento do processo de autorregulação da aprendizagem, pode-se afirmar que, na fase do Planejamento, as respostas dos estudantes caracterizaram-se pela antecipação do tempo necessário, estabelecimento de metas mais concretas e maior motivação para organizar o tempo. $\mathrm{Na}$ fase da execução, observaram-se respostas relacionadas ao estabelecimento de critérios para anotar e estudar, à busca de métodos e iniciativa para estudar, à administração do tempo, à divisão de grandes problemas para facilitar a resolução, além de respostas que relatavam a ação de conseguir evitar a preguiça e a procrastinação e ter o controle/evitação dos distratores. Quanto à fase da avaliação, os alunos relataram refletir acerca dos resultados do estudo e das suas próprias ações.

Para ilustração do que foi compreendido como fortalecimento do processo da ARA, relatos de estudantes serão exibidos. Aluna I. afirmou que a oficina " [...] me ajudou a enxergar que a melhora no meu desempenho acadêmico depende apenas de mim mesmo, do meu planejamento, da minha execução e autoavaliação" (Estatística); o aluno J. disse que "[...] agora sempre planejo minhas tarefas antes de executá-las, e pude observar melhoras no meu ritmo de estudos. Além de melhorar nas anotações e resumo, dada a maior facilidade em lê-los e entende-los" (Engenharia Mecânica).

Como consequência do fortalecimento da ARA, os alunos passaram a perceber o processo de estudo como mais dinâmico e útil, pois afirmaram que conseguiram desenvolver melhor as atividades em função do uso de estratégias de aprendizagem apropriadas às tarefas acadêmicas. Além disso, diferentes alunos afirmaram que a participação na oficina permitiu ganhos no desempenho acadêmico, seja por ler mais livros (K., Ciências Sociais), por estudar durante o semestre e não apenas durante a época de provas (J., Engenharia Mecânica) e pela aprovação em uma disciplina na qual já tinha sido reprovada (C., Física/ Matemática/Matemática Aplicada e Computacional).

\section{Considerações Finais}

A partir dos resultados quantitativos e qualitativos, pode-se dizer que foram obtidas evidências favoráveis para a eficácia do programa Cartas do Gervásio ao seu umbigo no que se refere à promoção da ARA de ingressantes em uma universidade pública brasileira. $\mathrm{O}$ programa impactou de maneira positiva os processos de estudo e aprendizagem dos ingressantes, possibilitando competências de natureza instrumental, interpessoal e sistêmica.

As competências de natureza instrumental dizem respeito à capacidade de analisar e sintetizar a informação; procurar e combinar informação de fontes diversas; organizar, planejar e programar tarefas no tempo, além de conhecer e exercitar estratégias de tomada de decisão. A competência interpessoal diz respeito ao trabalho em equipe e à importância do papel de cada um no produto final. A competência sistêmica implica a aplicação do conhecimento na prática; saber investigar e aprender; ter capacidade de adaptação a novas situações com soluções divergentes; trabalho autônomo e independente; e necessidade do empenho pessoal para alcançar sucesso (Nunes, 2009; Rosário \& Polydoro, 2014).

O programa "Cartas do Gervásio ao seu umbigo" deve ser compreendido e implementado tendo como referência seus pilares: narrativa, modelação e modelo teórico (Rosário et al., 2012; Rosário \& Polydoro, 2014). Especial atenção deve ser dada ao seu arcabouço teórico, a fim de garantir uma intervenção eficiente por parte do profissional que pretende aplicá-lo, primeiro, para compreender a estrutura do programa e, segundo, para ter segurança ao decidir quais atividades aplicar ou quais atividades inserir a partir do propósito estabelecido.

Associado a isso, faz-se necessário que o profissional tenha clareza, por exemplo, de que o desenvolvimento da competência autorregulatória não é linear, mas que algumas etapas podem ser seguidas para atingi-la (Rosário et al., 2012; Zimmerman, 2000). Além disso, para promover a ARA de maneira eficaz, devese considerar a(s) demanda(s) e/ou característica(s) do curso de graduação dos alunos (Hofer; Yu, \& Pintrich, 1998, Simpson et al., 1997), como eles pensam (Mullen, 2011), e as variáveis pessoais, como idade e histórico escolar anterior ao ingresso na educação superior.

Entende-se que o programa pode ser oferecido em atividades de recepção ao calouro (Carta 1), bem como com temas mais específicos, a depender do período do semestre, como a ansiedade frente às provas (Carta 12) (Dantas et al., 2017). Essas possibilidades se somam à opção do programa ser oferecido em serviços de apoio ao estudante tanto na modalidade grupal como na individual, para ingressantes e veteranos, tanto em formato 
de disciplina como em justaposição curricular (Pelissoni, 2016).

Em decorrência dos resultados desta pesquisa e da necessidade de atender alunos que estão em diferentes momentos de sua formação, a oficina "Como estudar melhor agora que estou na Universidade?", passou a ser oferecida como um programa regular do setor de orientação educacional do serviço de apoio ao estudante da universidade. Para isso, foram realizados ajustes na sequência das cartas e atividades propostas desde a sua implantação em 2011, conforme descrito por Dantas et al. (2017).

Apesar de as implicações desta pesquisa serem, em sua maioria, positivas, algumas limitações devem ser anunciadas. A primeira limitação refere-se ao número de participantes, à medida que a quantidade de estudantes sofreu impacto ao adotar-se o critério de presença em todos os seis encontros e, segunda, à falta de um grupo controle que pudesse oferecer referência para a análise dos dados obtidos no grupo que participou da oficina e, terceira, realizar uma avaliação de seguimento para aferir o impacto a médio e longo prazo na vida acadêmica do ingressante.

No que se refere às medidas de autorrelato, segue a quarta limitação, ao considerar que as médias obtidas no pré-teste, na maioria dos instrumentos, indicavam certo conhecimento de estratégias de aprendizagem, bem como o reconhecimento de alguma delas no processo de aprendizagem por parte dos ingressantes que optaram por realizar a atividade não obrigatória. Esses dados sugerem certo grau de autorregulação da aprendizagem dos alunos que se inscreveram, voluntariamente, numa atividade extracurricular, na hora do almoço, mediante ao título "Como estudar melhor agora que estou na Universidade?”, indicando que alunos com dificuldades mais severas ou não percebidas não foram atingidos. $O$ fato de essa oficina ser inserida no histórico curricular desse aluno pode vir a justificar a maior participação de ingressantes por incentivar a participação em uma disciplina não vinculada diretamente ao seu curso (Pelissoni, 2016).

Ainda referente às medidas de autorrelato, os resultados favoráveis ao pós-teste podem ser justificados pela familiaridade com os instrumentos na avaliação do pré-teste, bem como por estarem mais conscientes do que era esperado mediante a sua participação na oficina. Nesse sentido, entende-se que medidas de desempenho acadêmico podem ser outra fonte de informação para se aferir a eficácia de programas educacionais como exibido por Pelissoni (2016).
Apesar das limitações sinalizadas, este trabalho endossa a importância de se investir na promoção da autorregulação da aprendizagem dos estudantes da educação superior por diferentes meios. Diante da flexibilidade de uso e das evidências de eficácia da promoção da ARA por meio do programa "Cartas do Gervásio ao seu umbigo", considera-se que pode ser uma proposta a ser implantada tanto em serviços de apoio ao estudante por psicólogos escolares e pedagogos quanto em disciplinas por professores. Esse programa apresenta suporte teórico adequado, estratégias autorregulatórias, metodologia da narrativa e plasticidade de manuseio, os quais favorecem a absorção dos conhecimentos de novas estratégias, o autoconhecimento e, por sua vez, o desenvolvimento para autorregular a aprendizagem, o que vai ao encontro de alguns objetivos da educação superior brasileira (Brasil, 1996) e da aprendizagem ao longo da vida (Comissão Europeia [CE], 2006).

\section{Referências}

Bandura, A. (1986). Social foundations of thought and action: A social cognitive theory. Englewood cliffs, NJ: Prentice-Hall.

Bandura, A. (1991). Social Cognitive Theory of SelfRegulation. Organizational Behavior And Human Decision Processes, 50, 248-287. Recuperado de https://web.stanford.edu/dept/psychology/bandura/pajares/Bandura1991OBHDP.pd

Bandura, A. (2008). A evolução da Teoria social cognitiva. In Bandura, A. Azzi, R. G. \& Polydoro, S. A. J. Teoria Social Cognitiva: conceitos básicos (p. 15-41). Porto Alegre, RS: Artmed.

Campbell, D.T., \& Stanley, J.C. (1979). Delineamentos experimentais e quase-experimentais de pesquisa. Tradução de R. A. T. Di Dio. São Paulo: EPU-EDUSP.

Cerezo, R. (2010). Autorregulácion del aprendizaje en el ámbito universitario: Por qué esto y por qué abora? (Tese de Doutorado). Universidade de Oviedo, Oviedo, Espanha.

Dantas, M. A., Pelissoni, A. M., Freitas-Salgado, F.A., Polydoro, S. P. J., \& Rosário, P. (2017). Intervenção em autorregulação da Aprendizagem no ensino superior: Proposição e desdobramentos: Volume III. Em S. A. J. Polydoro (Ed.), Promoção da Autorregulação da aprendizagem: contribuiçoes da teoria social cognitiva (p. 77- 87). Porto Alegre, RS: Letra1.

Psico-USF, Bragança Paulista, v. 23, n. 4, p. 667-679, out./ dez. 2018 
Emílio, E. R. V., \& Polydoro, S. A. J. (2017). Autorregulação da Aprendizagem: Fundamentos e implicações no contexto educativo: Volume III. Em S. A. J. Polydoro (org.), Promoşão da Autorregulação da aprendizagem: contribuicões da teoria social cognitiva (p. 19 - 32). Porto Alegre, RS: Letra1.

Fabriz, S., Ewijk, C. D., Poarch, G., \& Büttner, G. (2014). Fostering self-monitoring of university students by means of a standardized learning journal a longitudinal study with process analyses. Eur J Psychol Educ, 29, 239-255. doi: 10.1007/ s10212-013-0196-z

Freitas-Salgado, F. A. (2013) Autorregulação da aprendizagem: Intervenção com alunos ingressantes do ensino superior (tese de doutorado). Universidade Estadual de Campinas - Unicamp, Campinas, São Paulo.

Hattie, J., Biggs, J., \& Purdie, N. (1996). Effects of learning skills interventions on student learning: $\mathrm{A}$ meta analysis. Review of Educational Research, 66(2), 99-136. doi: 10.3102/00346543066002099.

Hofer, B. K., Yu, S. L., \& Pintrich, P. (1998). Teaching college students to be self-regulated learners. Em Schunk, D., Zimmerman, B. (Eds.). Self-regulated learning: from teaching to self-reflective practice (p. 57-85). New York: The Guilford Press.

Khosa, D. K., \& Violet S. E. (2013). Promoting effective collaborative case-based learning at university: A metacognitive intervention. Studies in Higher Education, 38(6), 870- 889. doi:10.1080/03075079.201 1.604409 .

Lan, W. Y. (1998). Teaching self-monitoring skills in statistics. Em Schunk, D. \& Zimmerman, B. (Eds.), Self-regulated learning: from teaching to self-reflective practice (p. 86-105). New York: The Guilford Press.

Magalhães, C. M. S. (2009). A experiência do estudar na Universidade: Perspectivas auto-regulatórias de alunos de insucesso no $1^{\circ}$ ano (dissertação de mestrado). Universidade do Minho, Portugal.

Magalhães, C. R. (2012). A autorregulação da aprendizagem em Programa Institucional de Acolhimento e Suporte ao aluno universitário: Os professores como parceiros. Cadernos de Eeducaşão, 42, 143-167. Recuperado de https://periodicos.ufpel.edu.br/ojs2/ index.php/caduc/article/viewFile/2152/1969

Nunes, T. (2009). Programa de Promoção de Competências de Aprendizagem em Alunos do $1^{\circ}$ Ano do Ensino Superior: um estudo de caso com alunos de insucesso acadêmico (dissertação de mestrado). Universidade do Minho, Portugal.

Núñez, J. C., Cerezo, R., Bernardo, A., Rosário, P., Valle, A., Fernandez, E., \& Suárez, N. (2011). Implementation of training programs in self-regulated learning strategies in Moodle format: Results of a experience in higher education. Psicothema, 23(2), 274-281. Recuperado de http://www.psicothema. com/pdf/3882.pdf

Pajares, F., \& Olaz, F. (2008). Teoria social cognitiva e autoeficácia: Uma visão geral. Em A. Bandura; R. G. Azzi \& S. A. J. Polydoro (Eds.). Teoria social Cognitiva: conceitos básicos (p. 97-114). Porto alegre, RS: Artmed.

Parres, R., \& Flores, R. (2011). Experiencia educativa em arte visual diseñada bajo un modelo de autorregulación del aprendizaje con estudiantes universitarios. Revista Mexicana de Investigación Educativa, 16(49), 597-624. Recuperado de http://www. redalyc.org/articulo.oa?id=14018533012

Pelissoni, A. M. S. (2016). Eficácia de um programa híbrido de promoção da autorregulação da aprendizagem para estudantes do ensino superior (tese de doutorado). Unicamp, Campinas, São Paulo.

Pelissoni, A. M. S., \& Polydoro, S. A. J. (2017). Programas de promoção da autorregulação da Aprendizagem: Volume III. Em S. A. J. Polydoro (Eds.), Promoção da Autorregulação da aprendizagem: contribuiçoes da teoria social cognitiva (p. 33-44). Porto Alegre, RS: Letra1.

Pina, F. H., Rosário, P., Tejada, J. D. C., Clares, P.M., \& Lara, E. R. (2006). Promoción del aprendizaje estratégico y competencias de aprendizaje en estudiantes de primero de universidad: evaluación de una intervención. Revista de Investigacion Educativa, 24(2), 615-632. Recuperado de http://revistas. um.es/rie/article/view/97221/93351.

Pina, F. H., Rosário, P. J. S. L. F., \& Tejada, J. D. C. S. (2008). Impacto de un programa de autorregulácion del apredizaje en estudiantes de Grado. Revista de Educácion, 353, 571-588. Recuperado de http:// www.revistaeducacion.educacion.es/re353/ re353_21.pdf

Polydoro, S. A. J., \& Guerreiro-Casanova, D. C. (2010). Escala de autoeficácia na formação superior: Construção e estudo de validação. 
Avaliação Psicológica, 9(2). Recuperado de <http:// pepsic.bvsalud.org/scielo.php?script $=$ sci_ arttext\&pid=S1677-04712010000200011\&lng=pt \&nrm $=$ iso $>$.

Polydoro, S. A. J., Rosário, P.; Sampaio, R. K., \& Freitas, F. A. (2011). Sucesso no ensino superior e variáveis envolvidas. Em CONPE - Congresso Nacional de Psicologia Escolar e Educacional: Caminhos trilhados, caminhos a percorrer, 10, Maringá. Recuperado de https://abrapee.files.wordpress. com/2012/02/conpe-trabalhos-completos-anais_x-conpe-final.pdf.

Rosário, P., Núñez, J. C., \& González-Pienda, J. (2007). Autorregulação em crianças sub 10: Projecto Sarilhos do Amarelo. Portugal: Porto Editora.

Rosário, P., Mourão, R., Núñez, J. C., González-Pienda, J. A., Solano, P., \& Valle, A. (2007). Eficácia de un programa instruccional para la mejora de procesos y estrategias de aprendizaje en la enseñanza superior. Psicothema, 19(3), 422-427. Recuperado de http://www.psicothema.com/pdf/3380.pdf

Rosário, P. (2009a). Inventário de Processos de auto-regulação da Aprendizagem - Universidade. Versão para investigação. Universidade do Minho.

Rosário, P. (2009b). Questionário de Auto-eficácia e instrumentalidade da Autorregulação da Aprendizagem. Versão para investigação. Universidade do Minho.

Rosário, P., Mourão, R., Núñez, J. C., \& González-Pienda, J. A. (2010a). Promoting first-year students' Learning Strategies Through Insctructional Narratives. Em Nutt, D. \& Calderon, D. (Eds.) International perspectives on the first-year experience in higher education. Teesside University, Série 52.

Rosário, P., Nunes, T., Magalhães, C., Rodrigues, A., Pinto, R., \& Ferreira, R. (2010b). Processos de autorregulação da aprendizagem em alunos com insucesso no 1 ano de Universidade. Psicologia Escolar e Educacional, 14(2), 349-358. Recuperado de http://www.scielo.br/pdf/pee/v14n2/a17v14n2

Rosário, P., Núñez, J. C., González-Pienda, J., Valle, A., Trigo, L., \& Guimarães, C. (2010c). Enhancing self-regulation and approaches to learning in firstyear college students: A narrative-based program assessed in the Iberian Peninsula. European Journal Psychology Education, 25(4), 411-428. doi: 10.1007/ s10212-010-0020-y
Rosário, P., Polydoro, S. A. J., Fuentes, S., \& Gaeta, M. L. (2012). Programas de promoção da autorregulação ao longo da escolaridade: Estórias-ferramenta como motor da aprendizagem. Em Simão, A. M. V., Autorregulação da aprendizagem e narrativas autobiográficas: epistemologia e práticas (p. 179-207). Rio Grande do Sul: EDUFRN, EDUNEB, EDIPUCRS.

Rosário, P., Núñez, J. C., \& González-Pienda, J. (2012). Cartas do Gervásio ao seu Umbigo: Comprometer-se com o Estudar na Educação Superior. São Paulo: Almedina Editores.

Rosário, P., \& Polydoro, S. A. J. (2014). Capitanear o aprender: Promoção da autorregulação da aprendizagem no contexto escolar. São Paulo: Casa do Psicólogo.

Sánchez, J. M. R. (2004). Procedimiento de aprendizaje autorregulado para universtiarios: La 'estrategia de lectura significativa de textos’. Revista Electrónica de Investigácion Psicoeducativa, 2(1), 113-132. Recuperado de http://www.investigacionpsicopedagogica. org/revista/articulos/3/espannol/Art_3_33.pdf

SAS (Statistical Analysis System) - System for Windows, versão 8.02. SAS Institute Inc, 1999-2001, Cary, NC, USA, 2001.

Schloemer, P., \& Brenan, K. (2006). From students to learners: Developing self-regulated learning. Journal of Education For Business, 81-87. doi: 10.3200/ JOEB.82.2.81-87

Schunk, D. (2001). Social Cognitive Theory and Selfregulated Learning. Em Schunk, D.; Zimmerman, B. (Eds.) Self-regulated learning and academic achievement: Theoretical perspectives (p. 125-151). 2.ed. New York: Lawrence Erlbaum Associates.

Seco, G. M., Pereira, A. P., Santos, I. C., Filipe, L. A., \& Alves, S. (2008). Promoção de estratégias de aprendizagem: contributos do serviço de apoio ao estudante (SAPE) do Instituto Politécnico de Leiria (IPL). INFAD Revista de Psicologia, 1, 295-304. Recuperado de http://hdl.handle.net/10400.8/59

Simpson, M. L., Hynd, C. R., Nist, S. L., \& Burrell, K. I. (1997). College academic assistance programs and practices. Educational Psychology Review, 9(1), 39-87. doi: 10.1023/A:1024733706115

Solano, P. (2006). Elaboración y evaluácion de um programa de mejora de la competência e estratégias de autorregulación (tese de doutorado). Universidade de Oviedo, Oviedo, Espanha. 
Vanderstoep, S. W., \& Pintrich, P. (2008). Learning to Learn: The skill and will of college success. 2. ed. New Jersey; Ohio: Upper Saddle River; Columbus.

Zimmerman, B. J. (1989). A Social Cognitive view of Self-Regulated Academic Learning. Journal of Educational Psychology, 81(3), 329-339. doi: 10.1037/0022-0663.81.3.329.

Zimmerman, B. J. (1998). Developing self-fulfilling cycles of academic regulation: an analysis of exemplary instructional models. Em Schunk, D.; Zimmerman, B. (Eds.). Self-regulated learning: from teaching to self-reflective practice (p. 1-19). New York: The Guilford Press.

Zimmerman, B. J. (2000). Attaining self-regulation: A social-cognitive perspective. Em Boekaerts, M., Pintrich, P., \& Zeidner, M. (Eds.) Self-regulation:
Theory, research, and applications (p. 13-39). Orlando: FL7 Academic Press.

Zimmerman, B. J. (2002). Becoming a self-regulated learner: An overview. Theory into Pratice, 41(2), 6470. doi: 10.1207/s15430421tip4102_2

Zimmerman, B. J., Bonner, S., \& Kovach, R.. (2008). Developing self-regulated learners: Beyond achievement to self-efficacy. Washington, DC: American Psyhcological Association.

Zimmerman, B. J., \& Schunk, D. H. (2011). Self-regulated and Performance: An introduction and an overview. Em Zimmerman, B., \& Schunk, D. H. Handbook of self-regulation of learning and performance (p. 1-12). New York; London: Routledge: Taylor; Francis Group.
Recebido em: 24-06-2017

Reformulado em: 21-11-2017

Aprovado em: 22-03-2018

Sobre os autores:

Fernanda Andrade de Freitas Salgado é mestra em Avaliação Psicológica pela Universidade São Francisco (2004), doutora em Educação pela Universidade Estadual de Campinas-UNICAMP (2013) e docente do curso de Psicologia do Centro universitário nossa Senhora do Patrocínio - CEUNSP.

E-mail: fisafreitas@gmail.com

Soely Aparecida Jorge Polydoro é mestra em Psicologia escolar pela Pontifícia Universidade Católica de Campinas (1995), doutora em Educação pela Universidade Estadual de Campinas-UNICAMP (2000), atualmente é professora da UNICAMP, do departamento de Psicologia Educacional e vice-líder do Grupo de Pesquisa Psicologia Educação Superior-Faculdade de Educação e membro do GT Pesquisa em Avaliação Psicológica da Associação Nacional de Pesquisa e Pós-graduação em Psicologia.

E-mail: soleypolydoro@gmail.com

Pedro Rosário é doutor em Psicologia pela Universidade do Minho, professor associado com agregação na mesma universidade, vinculado ao Departamento de Psicologia Aplicada da Escola de Psicologia - Portugal, e investigador principal do grupo GUIA - Grupo universitário de Investigação em Autorregulação da Aprendizagem. Disponível em http://www.guia-psi.com/

E-mail: prosario@psi.uminho.pt

Contato com os autores:

Faculdade de Educação da Unicamp

Av. Bertrand Russell, 801, Cidade Universitária "Zeferino Vaz"

Campinas-SP, Brasil

CEP: 13083-865

Psico-USF, Bragança Paulista, v. 23, n. 4, p. 667-679, out./ dez: 2018 